Modeling, Identification and Control, Vol. 28, No. 3, 2007, pp. 69-79

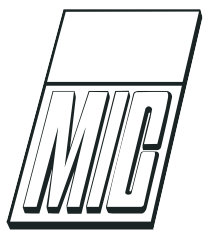

\title{
Nonlinear Model-Based Control of Unstable Wells
}

\author{
Glenn-Ole Kaasa ${ }^{1}$ Vidar Alstad $^{1}$ Jing Zhou ${ }^{2}$ Ole Morten Aamo ${ }^{2}$ \\ ${ }^{1}$ Hydro Oil \& Energy, Research Centre, N-3908 Porsgrunn, Norway. E-mail: \{Glenn-Ole.Kaasa, Vidar.Alstad\} \\ Qhydro.com \\ ${ }^{2}$ Department of Engineering Cybernetics, Norwegian University of Science and Technology, N-7491 Trondheim, \\ Norway. E-mail: jing.zhou@itk.ntnu.no, aamo@ntnu.no
}

\begin{abstract}
This paper illustrates the potential of nonlinear model-based control applied for stabilization of unstable flow in oil wells. A simple empirical model is developed that describes the qualitative behavior of the downhole pressure during severe riser slugging. A nonlinear controller is designed by an integrator backstepping approach, and stabilization for open-loop unstable pressure setpoints is demonstrated. The proposed backstepping controller is shown in simulations to perform better than PI and PD controllers for low pressure setpoints, and is in addition easier to tune. Operation at a low pressure setpoint is desirable since it corresponds to a high production flow rate. The simulation results are presented to illustrate the effectiveness of proposed control scheme.
\end{abstract}

Keywords: Nonlinear control, slugging, backstepping, stabilization.

\section{Introduction}

In tail production, i.e. oil production from mature fields where the reservoir is about to be drained, unstable multiphase flow from wells - or severe slugging - is an increasing problem. In particular, unstable flow causes reduced production and oil recovery as the well must be choked down for the downstream processing equipment on the platforms to be able to handle the resulting variations in liquid and gas flow rates.

Active control of the production choke at the well head can be used to stabilize or reduce these instabilities. Conventionally, this is done by applying PI control to a measured downhole pressure to stabilize this at a specified set point, thus stabilizing the flow. For wells, however, PI control is often insufficient: Either it is not robust and requires frequent re-tuning, or it does not achieve proper stabilization at all.

Consequently, improved methods for stabilization of slugging wells have significant potential for increased production and recovery. Typically, there is dynamics between the choke input and the measured pressure such that the PI controller reacts too late to compensate the instabilities in the flow. In these cases, a simple model may be used to develop a model-based control law which more intelligently counteracts the destabilizing mechanisms in unstable flow, i.e., balances the pressure oscillations in the well. This work presents some preliminary efforts in that direction.

Based on the observation that the system behaves like a stable limit cycle, a simple empirical model is developed that describes the main qualitative behavior of the downhole pressure during slugging. This model is then used to perform a preliminary analysis of nonlinear model-based control applied to stabilize the system.

The paper is organized as follows: First, in Section 2, we provide a background description and a brief literature review of unstable multiphase flow - or slugging, and how this instability is conventionally handled. In Section 3 we propose a simple empirical model that describes the main qualitative properties of this unstable phenomena, to be used for analysis and design of a model-based control law. In Section 4, we illus- 
trate the design of a nonlinear control law by integrator backstepping, and in Section 5, we compare the performance of the nonlinear controller with the conventional PI-controller and a PD-controller. Finally, in Section 6 , we draw some brief conclusions.

\section{Unstable multiphase flow}

Multiphase flow instabilities can be present in all phases of the lifetime of a field, however, the likelihood for multiphase flow instabilities increases when entering tail production. In tail production, the reservoir pressure typically decrease, yielding lower fluid velocities and increased gas-liquid ratio (GLR). In addition, the water cut (water fraction) tends to increase, making the liquid denser. Low velocities and increased liquid density, yield a potential for unstable flow in wells and pipelines due to gravity dominated pressure loss. In many cases, the wells and production lines enter a slug flow regime where liquid slugs are followed by gas pockets yielding large oscillations in the flow rate and phase distribution as seen from the outlet of the pipe/well. This alternating flow regime, referred to as severe slugging, poses a serious operational challenge for the downstream process. In typical offshore production processes there is no buffer capacity to handle the flow variations due to severe slugging, resulting in poor separation and process utilization, and in worst case, shut-down of the process. In addition, the trend in offshore developments is to tie in smaller discoveries to existing infrastructure, yielding longer transport distances with multiphase flow. The flow assurance strategy including the control solutions must then be carefully selected including the control solution in order for efficient and safe transportation of the fluids to the separation facility.

For unstable flow, several mechanisms can cause the instability depending on the geometry, fluids and process equipment. A description of slug flow and the underlying mechanisms for it can be found in Pickering et al. (2001).

Severe slugging in pipeline-riser systems has received much attention in the literature and in the industry. A schematic of the severe slugging cyclic behavior is shown in Figure 1, where the main phases of the formation of a slug is illustrated In the first sub-figure, liquid blocks the low point of the pipeline-riser system, preventing the gas from passing. Liquid flows from the riser and into the slug by gravity and causes the slug to grow and fill the riser. The pressure in the pipeline increases due to the inlet flow of gas and the increased liquid head. In the "slug production phase" the liquid slug has reached the top of the riser and flows into the separator. The pressure in the pipeline has steadily

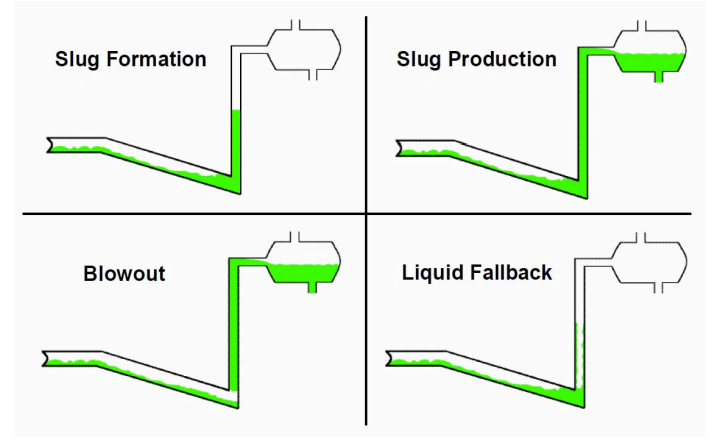

Figure 1: Schematics of the severe slug cycle in flowline riser systems Pickering et al. (2001)

increased and is now large enough to push the liquid slug out of the riser. When the tail of the liquid slug enters the riser, the pressure drops due to the reduced static head of the liquid column which causes the gas to expand and accelerate the "blow out phase". When the gas has left the riser, the velocities in the riser are too low to carry any liquid up the riser and the process starts over ("liquid fall-back phase").

Storkaas (2005) present a relatively simple first principle-based model which captures the main dynamics of a severe slugging flow regime (Storkaas et al., 2003) in pipeline-riser systems, referred to as riser slugging. The model is able to reproduce observed unstable flow for a particular test case, however, it is not straight forward to extend the model to other geometries, e.g. wells. Storkaas (2005) observed that when plotting the bottom hole pressure versus choke valve opening, the system is stable at low valve openings (fixed choking), while when increasing the choke valve opening above a certain value (the bifurcation point), the system starts to oscillate and enters a stable limit cycle.

Slugging is not restricted to pipeline-riser systems. Existing drilling technology makes it possible to drill long horizontal wells which yields a geometry that resembles a pipeline-riser geometry. Instabilities in gaslift wells (casing-heading instabilities) has been observed in practice (Jansen et al., 1999), and recent academic work on the phenomena can be found in Eikrem (2006) and Sinègre (2006). The instabilities in gas-lift wells are due to the interacting between the gas casing and production tubing, where the underlaying phenomena and solutions for stabilizing the casing-heading instability has been to a large extent solved. Unstable flow in production wells can be as severe for the production as terrain induced slugging in pipeline-riser systems, but the underlaying instability mechanisms are not fully understood (Dalsmo et al., 2002). 


\subsection{Slug modelling and prediction}

Several methods for predicting the stability of multiphase flow have been proposed. The simplest approach is to use flow maps which categorizes the different flow regimes as a function of superficial gas and liquid velocities. The flow maps vary significantly with flow conditions (such as pipeline geometry), such that experiments are necessary in order to obtain accurate predictions. Local methods such as Kelvin-Helmholtz stability criteria (Lin and Hanratty, 1986; Taitel and Dukler, 1975) can predict local flow regimes. However, for the overall dynamics of the pipeline, these methods are not suited. With respect to severe slugging, several conditions has been proposed (Bøe, 1986; Schmidt et al., 1985; Taitel, 1986). These criteria are based on steady state analysis which usually requires variables that are not readily available.

In order to predict severe slugging, a dynamic model of the pipe is necessary. An approach based on the dynamic DAE models of the system is presented in Zakarian (2000). The approach is based on a fixed geometry of a pipe and riser, where a criterion for the stability of the system is derived based on linear analysis. Based on a nonlinear analysis, it is shown that the severe slugging phenomenon is a hydrodynamic instability due to a supercritical Hopf bifurcation. The method has not been extended to other geometries, which limits its usefulness.

Predicting and modelling unstable flow in production wells is complex and the existing state-of-the-art model for multiphase flow (OLGA2000) is not capable of reproducing the instabilities observed for wells. In addition, the dynamics of the near well-bore reservoir region, is not satisfactorily understood, which complicates modelling further.

\subsection{Handling slugging}

When it comes to handling unstable slug flow, several remediation strategies has been proposed. These can be categorized as:

\subsubsection{Design modifications}

- Slug catcher: A solution which is robust is to use a slug catcher which will act as a buffer and as a first stage separator. This solution is acceptable for smaller hydrodynamic slugs $\left(10 \mathrm{~m}^{3}\right)$. For severe slugs (on the order of $100 \mathrm{~m}^{3}$ ) the required vessel will be excessive in size and weight for some development projects.

- Pipeline diameter: In the design phase pipe diameter can be optimized to avoid slugging. Small diameter pipes results in large fluid velocities. Typ- ically, hydrodynamic slugs are generated at high velocities and severe slugging is generated at low velocities. Finding a diameter that is acceptable for a wide range of flow rates can be difficult. In addition the increased pressure drop for a small diameter piping will increase the frictional pressure drop which is undesirable from a production perspective. Variable diameter pipes are one possible solution (e.g. a "velocity string" where a smaller pipe is inserted in the existing riser as a retrofit solution (Tengesdal et al., 2003)). No applications of this technology has been published in the literature.

- Homogenising multiphase flow: In order to break the intermittent flow, surfactants can be added (Wilkins et al., 2003). The effect of the surfactants on separation has a negative sideeffect which makes it less desirable.

- Separator control: The slug suppression system $\left(S^{3}\right)$ (Kovalev et al., 2003) install a small separator upstream the main separator and control the flow rate of oil and gas using the gas outlet valve in order to stabilize the flowline. Several successful installations has been published (Kovalev et al., 2004). The mini-separator can also be replaced with a larger diameter pipe with a gas outlet.

\subsubsection{Operational modifications}

- Fixed choking: By reducing the choke opening, the increased backpressure will eventually dominate the pressure drop in the riser and move the flow system into the stable region (Schmidt et al., 1980; Farghaly, 1987). In most cases, the increased backpressure resulting from reduced choke opening is unwanted as it lowers the production rate from the well.

- Active choke: Several publications describe the use of active feedback control in order to stabilize the flow (Henriot et al., 1999; Drengstig and Magndal, 2002; Molyneux et al., 2000; Dalsmo et al., 2002; Kinvig and Molyneux, 2001; Godhavn et al., 2005; Storkaas, 2005). The motivation for using active feedback control is that one can operate the pipeline/well in an unstable operating region, where the system is open-loop unstable.

- Gas lift: In systems which use gas lift for production enhancement, the gas lift flow rate can be used to move the system into an open-loop stable region. Typically, the gas lift capacity is a limited resource which in some cases makes it impossible to move the system into the stable region. 


\section{Modelling}

The oscillating behavior of the downhole pressure of a slugging well can be characterized as a stable limit cycle. Severe slugging exhibits qualitatively the same behavior as the slightly modified van der Pol equation

$$
\begin{aligned}
\dot{p} & =w \\
\dot{w} & =a_{1}(\beta-p)+a_{2}\left(\zeta-w^{2}\right) w
\end{aligned}
$$

where the states $p$ and $w$ are the down hole pressure in the well and its time derivative, respectively. The coefficients in (1)-(2) can be explained as follows.

- $\beta$ : steady state pressure.

- $a_{1}$ : frequency or stiffness of the system.

- $a_{2}, \zeta$ : local "degree of the stability/instability" and amplitude of the oscillation.

\subsection{The equilibrium downhole pressure $\beta$}

The equilibrium point $\left(p^{*}, w^{*}\right)$ of the system $(1)-(2)$ becomes

$$
\left[\begin{array}{l}
p^{*} \\
w^{*}
\end{array}\right]=\left[\begin{array}{l}
\beta \\
0
\end{array}\right],
$$

which means that the parameter $\beta$ is simply the equilibrium downhole pressure $p^{*}$. The equilibrium downhole pressure $p^{*}=\beta$ is given by

$$
\beta=\bar{\rho} g H+\Delta p_{f}+\Delta p_{c}+p_{0} .
$$

where $\bar{\rho} g H$ is the static head with $\bar{\rho}$ being the average density in the riser, $\Delta p_{f}$ the frictional pressure drop, $\Delta p_{c}$ the pressure drop over the production choke, and $p_{0}$ the pressure downstream the choke. For a given reservoir influx $w_{\text {res }}$, the differential pressure over the production choke is given by its flow characteristic according to

$$
\Delta p_{c}\left(w_{r e s}\right)=\frac{w_{r e s}^{2}}{\left(K_{c} u_{c}\right)^{2} \rho_{c}},
$$

where $\rho_{c}$ is the density upstream the choke, $u_{c}$ the choke opening, and $K_{c}$ the flow constant of the choke. Furthermore, the average density $\bar{\rho}\left(w_{\text {res }}\right)$ is a decreasing function of $w_{r e s}$ determined by the liquid holdup, and the frictional pressure drop $\Delta p_{f}\left(w_{r e s}\right)$ is like (4), an increasing function of $w_{\text {res }}$ according to

$$
\Delta p_{f}=K_{f} w_{r e s}^{2} .
$$

In the simplest case, we may assume constant influx $w_{\text {res }}$ such that $\beta$ can be given in the lumped form

$$
\beta(q)=b_{0}+b_{1} q
$$

where $b_{0}$ and $b_{1}$ are positive constants, and $q$ is proportional to the differential pressure $\Delta p_{c}$ at steady-state flow $w_{\text {res }}$. In Figure $2, \beta$ is plotted as a function of the choke opening.

\subsection{Local Degree of Stability/Instability $a_{2}, \zeta$}

The parameters $a_{2}$ and $\zeta$ are related to the amplitude of oscillation and stability properties of the fixed point. This can be seen by linearizing system (1)-(2) to get

$$
\begin{aligned}
\dot{\Delta} p & =\Delta \omega, \\
\dot{\Delta} \omega & =-a_{1} \Delta p+a_{2} \zeta \Delta \omega .
\end{aligned}
$$

The eigenvalues of the system are $\lambda=\frac{a_{2} \zeta \pm \sqrt{a_{2}^{2} \zeta^{2}-4 a_{1}}}{2}$, which means that (assuming $a_{1}>0$ and $a_{2}>0$ )

- $\zeta=0$, bifurcation point.

- $\zeta<0$, system is stable.

- $\zeta>0$, system is unstable.

In the simplest case, we may assume constant flow rates of liquid and gas from the reservoir. Then

$$
\zeta(q)=c_{0}-c_{1} q,
$$

where $c_{0} / c_{1}$ denotes the bifurcation point and $c_{0}, c_{1}$ are positive constants.

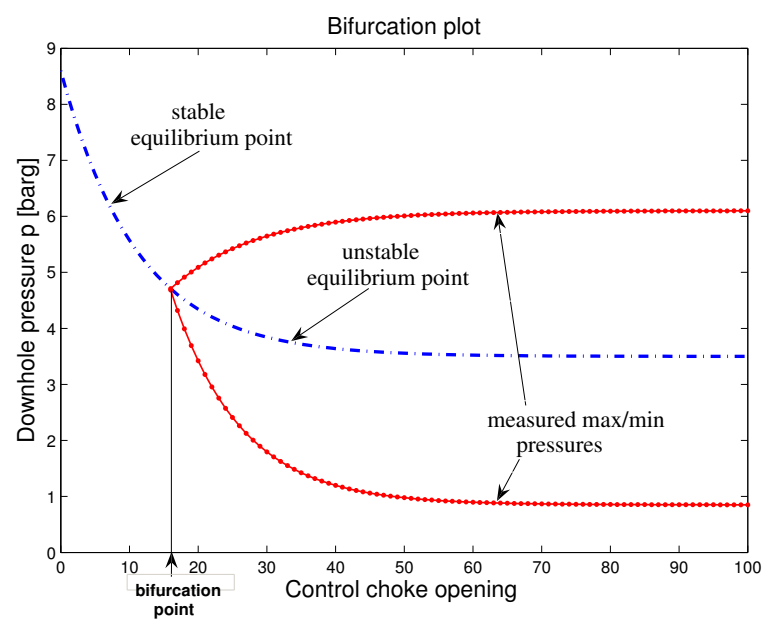

Figure 2: Bifurcation plot

\subsection{Transportation Delay}

The variable $q$ is related to the effect the differential pressure over the production choke has on the dynamics of (1)-(2). Due to transport delay in the well, a 
time-lag is expected between application of the control signal to the choke and seeing the effect in (1)-(2). This time-lag is modelled as follows

$$
\dot{q}=-\frac{1}{\tau} q+\frac{1}{\tau} \delta
$$

where $\delta$ represents the control input and is a strictly decreasing function of the production choke opening $u \in[0,1]$. Thus, when $\delta$ is computed, the actual control signal to apply to the choke is found by inverting $\delta(u)$. It is assumed that $\delta \rightarrow \infty$ as $u \rightarrow 0$, and that $\delta \geq$ $\delta_{\min } \geq 0$. Without loss of generality, we let $\delta_{\text {min }}=0$.

\subsection{Simplified Model of Riser Slugging}

Based on (5) and (8), the system dynamics (1)-(2) and (9) can be assembled into

$$
\begin{aligned}
\dot{p} & =w, \\
\dot{w} & =-a_{1} p+h(w)+g(w) q+a_{1} b_{0} \\
\dot{q} & =-\frac{1}{\tau} q+\frac{1}{\tau} \delta,
\end{aligned}
$$

where the functions $h$ and $g$ are defined as

$$
\begin{aligned}
h(w) & =a_{2} c_{0} w-a_{2} w^{3} \\
& =h_{0} w-h_{1} w^{3} \\
g(w) & =a_{1} b_{1}-a_{2} c_{1} w \\
& =g_{0}-g_{1} w .
\end{aligned}
$$

The positive constants $a_{i}, b_{i}$ and $c_{i}(i=1,2)$ are empirical parameters that are adjusted to produce the right behavior of the downhole pressure $p$.

The system (10)-(12) can capture some of the qualitative properties in the downhole pressure during riser slugging.

- Decreasing control gain: A characteristic property of riser slugging is that the static gain decreases with choke opening.

- Bifurcation: The model exhibits the characteristic bifurcation that occurs at a certain choke opening $c_{0} / c_{1}$, i.e., the steady-state response of the downhole pressure exhibits changes from a stable point when choke opening is smaller than $c_{0} / c_{1}$ to a stable limit cycle when choke opening is larger that $c_{0} / c_{1}$ (see Figure 2).

- Time lag: The transportation delay between a change in choke opening to the resulting change in downhole pressure $p$ is modeled by simple 1storder lag.

Our objective is to design a control law for the control input $\delta$ which stabilizes $p$ at the desired set-point $p_{\text {ref }}$.

\section{Controller Design}

In this section we design stabilizing controllers using backstepping. Thus, we iteratively look for a change of coordinates in the form

$$
\begin{aligned}
& z_{1}=p-p_{\text {ref }} \\
& z_{2}=w-\alpha_{w} \\
& z_{3}=q-\alpha_{q}
\end{aligned}
$$

and an accompanying Lyapunov function. The functions $\alpha_{w}$ and $\alpha_{q}$ are virtual controls to be determined.

\subsection{Control Scheme I}

Step 1 - virtual control law $\alpha_{w}$

From (10), (15) and (16), we obtain that

$$
\dot{z}_{1}=\alpha_{w}+z_{2}
$$

Then we design a virtual control law $\alpha_{w}$

$$
\alpha_{w}=-C_{1} z_{1} .
$$

The time-derivative of $U_{1}=\frac{1}{2} z_{1}^{2}$ becomes

$$
\dot{U}_{1}=-C_{1} z_{1}^{2}+z_{1} z_{2}
$$

Step 2 - virtual control law $\alpha_{q}$

We start by computing the time-derivative of $z_{2}$ using (11) and (15)-(17), obtaining

$$
\begin{aligned}
\dot{z}_{2}= & -a_{1}\left(z_{1}+p_{\text {ref }}-b_{0}\right)+h(w) \\
& +g(w) \alpha_{q}+g(w) z_{3}-\dot{\alpha}_{w} .
\end{aligned}
$$

If we for now ignore (14) and instead assume that $g(w) \geq g_{0}>0$, we may choose the virtual control $\alpha_{q}$ as

$$
\begin{aligned}
\alpha_{q}= & \frac{1}{g(w)}\left(-C_{2} z_{2}-z_{1}+a_{1}\left(z_{1}+p_{r e f}-b_{0}\right)\right. \\
& \left.-h(w)-a_{1} b_{0}+\dot{\alpha}_{w}\right)
\end{aligned}
$$

Consider the CLF

$$
U_{2}=U_{1}+\frac{1}{2} z_{2}^{2}
$$

The time derivative of $U_{2}$ is

$$
\dot{U}_{2}=-C_{1} z_{1}^{2}-C_{2} z_{2}^{2}+g(w) z_{2} z_{3} .
$$

Step 3 - Final control law $\delta$ 
The dynamics of $z_{3}$ is obtained as

$$
\begin{aligned}
\dot{z}_{3} & =\dot{q}-\dot{\alpha}_{q} \\
& =-\frac{1}{\tau} q+\frac{1}{\tau} \delta-\dot{\alpha}_{q} .
\end{aligned}
$$

Selecting

$$
\delta=-\tau C_{3} z_{3}-\tau g(w) z_{2}+\alpha_{q}+\tau \dot{\alpha}_{q},
$$

the derivative of the control Lyapunov function

$$
U_{3}=U_{2}+\frac{1}{2} z_{3}^{2}
$$

becomes

$$
\begin{aligned}
\dot{U}_{3} & =-C_{1} z_{1}^{2}+g(w) z_{2} z_{3}+z_{3}\left(-\frac{1}{\tau} q+\frac{1}{\tau} \delta-\dot{\alpha}_{q}\right) \\
& \leq-C_{1} z_{1}^{2}-C_{2} z_{2}^{2}-C_{3} z_{3}^{2}
\end{aligned}
$$

which proves that the equilibrium $\left(z_{1}, z_{2}, z_{3}\right)=0$ is globally exponentially stable, and in particular $p$ is regulated to the setpoint $p_{\text {ref }}$. The rate of convergence is adjustable through the constants $C_{1}, C_{2}$, and $C_{3}$, and we may in principle have any desirable performance of the system. The resulting control law is

$$
\begin{aligned}
& \delta(p, w, q) \\
= & -\tau C_{3} q-\tau g(w)\left(w+C_{1}\left(p-p_{\text {ref }}\right)\right) \\
& +\frac{1}{g^{2}(w)}\left[\tau \left(\left(C_{3}+1\right) g(w)-g^{\prime}(w)(h(w)+\right.\right. \\
& \left.\left.-a_{1}\left(p-b_{0}\right)+g(w) q\right)\right)\left(-\left(C_{1}+C_{2}\right) w-h(w)\right. \\
& \left.\left.-\left(C_{1} C_{2}+1-a_{1}\right)\left(p-p_{r e f}\right)+a_{1}\left(p_{r e f}-b_{0}\right)\right)\right] \\
& -\frac{1}{g(w)}\left[\tau ( C _ { 1 } + C _ { 2 } + h ^ { \prime } ( w ) ) \left(-a_{1}\left(p-b_{0}\right)\right.\right. \\
& \left.+h(w)+g(w) q)+\tau\left(C_{1} C_{2}+1-a_{1}\right) w\right]
\end{aligned}
$$

Remark 1. We refer to this choice of $\alpha_{q}$ as an exact cancelling design because we simply cancel existing dynamics and replace it with some desirable linear feedback terms: $-C_{1} z_{1}$ and $-C_{2} z_{2}$. Note that this design is not necessarily the best choice of control law because stabilizing nonlinearities may be cancelled, potentially wasting control effort, losing robustness to modelling errors, and making the control law overly complicated. As can be seen in (28), the controller becomes quite complicated as a result of the virtual controls and their time derivatives occuring in it. It is desirable to obtain a simpler control law, which is possible if simple virtual controls can be found by avoiding cancellation of useful nonlinearities.

\subsection{Control Scheme II}

The design of the previous Section is a straight forward application of the backstepping technique. However, it ignores (14) as well as the fact that the control input $\delta$ saturates at 0 . In this section, a better control law will be obtained by exploiting the structure of the system in terms of the specific choices for $h(w)$ and $g(w)$ in (13)(14), and the flexibility of the backstepping procedure in selecting virtual control laws.

By inspection of the second step of backstepping in the previous section, we recognize that the terms $-h_{1} w^{3}$ and $-g_{1} w q$ are expected to be stabilizing, since physically $q \geq 0$. Hence, cancelling these terms is not necessary at this point in the design. Substituting (13) and (14) into (20), and selecting $\alpha_{w}=0$ and

$$
\begin{aligned}
\alpha_{q} & =-\frac{C_{2}+h_{0}}{g_{0}} z_{2}+\frac{a_{1}}{g_{0}}\left(p_{\text {ref }}-b_{0}\right), \\
U_{2} & =\frac{a_{1}}{2} z_{2}^{2}+\frac{1}{2} z_{2}^{2},
\end{aligned}
$$

gives

$$
\dot{U}_{2}=-\left(C_{2}+g_{1} q\right) z_{2}^{2}-h_{1} z_{2}^{4}+g_{0} z_{2} z_{3} .
$$

Here, we notice that the $z_{1} z_{2}$-cross-term was cancelled, due to the particular choice of $U_{2}$ and $\alpha_{w}$. The stabilizing terms $-h_{1} z_{2}^{3}$ and $-g_{1} \alpha_{q} z_{2}$ increase negativity of $\dot{U}_{2}$, and need not be compensated for at this point. Consider now the CLF

$$
U_{3}=U_{2}+\frac{1}{2} z_{3}^{2}
$$

It's time derivative is

$$
\begin{aligned}
\dot{U}_{3}= & -\left(C_{2}+g_{1} q\right) z_{2}^{2}-h_{1} z_{2}^{4} \\
& +z_{3}\left(g_{0} z_{2}-\frac{1}{\tau} q+\frac{1}{\tau} \delta-\dot{\alpha_{q}}\right),
\end{aligned}
$$

and we may select

$$
\delta=-\tau C_{3} z_{3}-\tau g_{0} z_{2}+q+\tau \dot{\alpha}_{q}
$$

to obtain

$$
\dot{U}_{3}=-\left(C_{2}+g_{1} q\right) z_{2}^{2}-h_{1} z_{2}^{4}-C_{3} z_{3}^{2} .
$$

LaSalle's invariance principle now implies that the origin is asymptotically stable. The following result formalizes this, and in addition takes saturation of $\delta$ into account.

Theorem 1. Let $p_{\text {ref }}>b_{0}, C_{2}>0$ and $C_{3}>0$. Then the equilibrium $x_{\text {ref }}=\left(p_{\text {ref }}, 0, a_{1}\left(p_{\text {ref }}-b_{0}\right) / g_{0}\right)$ of system (10)-(12) in closed loop with the saturated 
control

$$
\delta=\max \left\{0, \delta_{a}\right\}
$$

where

$$
\begin{aligned}
& \delta_{a}(p, w, q) \\
= & \frac{\left(C_{2}+h_{0}\right)}{g_{0}}\left[\tau a_{1} p(t)-\tau\left(C_{3}+h_{0}\right) w(t)\right. \\
& \left.+\tau h_{1} w^{3}(t)+\tau g_{1} w(t) q(t)-\tau a_{1} b_{0}\right] \\
& -\tau g_{0} w(t)+\left(1-\tau\left(h_{0}+C_{2}+C_{3}\right)\right) q(t) \\
& +\frac{a_{1} \tau C_{3}}{g_{0}}\left(p_{\text {ref }}-b_{0}\right)
\end{aligned}
$$

is asymptotically stable. If

$$
C_{2} \leq \frac{1}{2 \tau}-h_{0}
$$

then the set

$$
\mathcal{A}=\left\{(p, w, q) \mid p \geq \underline{p}_{0}, \underline{w}_{0} \leq w \leq \bar{w}_{0}, q \geq 0\right\}
$$

where

$$
\begin{aligned}
& \underline{p}_{0}=\frac{1}{4}\left(3 p_{\text {ref }}+b_{0}\right) \\
& \underline{w}_{0}=-\min \left\{\frac{g_{0}}{2 \tau g_{1}\left(C_{2}+h_{0}\right)}, \sqrt[3]{\frac{a_{1}\left(p_{r e f}-b_{0}\right)}{4 h_{1}}}\right\} \\
& \bar{w}_{0}=\frac{a_{1}\left(C_{2}+h_{0}\right)\left(p_{r e f}-b_{0}\right)}{4\left(g_{0}^{2}+C_{2} h_{0}+h_{0}^{2}\right)}
\end{aligned}
$$

is contained in the region of attraction of $x_{r e f}$.

Proof: The condition $p_{\text {ref }}>b_{0}$ ensures that $\delta_{a}>0$ at the equilibrium $z=\left(z_{1}, z_{2}, z_{3}\right)=0$. Thus, in view of (32) and (35), there exists a constant $c>0$ such that $D=\left\{z \mid U_{3}(z)<c\right\}$ is positively invariant and $\delta_{a}>0$ and $q(t)>0$ for all $z \in D$. Thus, from (35) we have

$$
\dot{U}_{3} \leq-C_{2} z_{2}^{2}-C_{3} z_{3}^{2}
$$

in $D$. Furthermore, only $z(t) \equiv 0$ stays forever in $S=\left\{z \in D \mid \dot{U}_{3}=0\right\}$ since $\dot{z}_{2}=-a_{1} z_{1}$ for $z \in S$. Therefore, by Corollary 4.1 of Khalil (2002) $z=0$ is asymptotically stable.

The estimate of the region of attraction is obtained by analyzing $\dot{U}_{3}$ when $\delta$ is saturated as follows. From the condition $q(0) \geq 0$, equation (12), and the fact that $\delta(t) \geq 0$ for all $t>0$, we have that $q(t) \geq 0$ for all $t>0$. So, from (33) we have

$$
\dot{U}_{3} \leq-C_{2} z_{2}^{2}+z_{3}\left(g_{0} z_{2}-\frac{1}{\tau} q+\frac{1}{\tau} \delta-\dot{\alpha_{q}}\right) .
$$

Now, let $\delta_{a}<0$. Then, $\delta=0$,

$$
\dot{z}_{3}=-\frac{1}{\tau} q-\dot{\alpha}_{q}
$$

and the derivative of $U_{3}$ satisfies

$$
\dot{U}_{3} \leq-C_{2} z_{2}^{2}+z_{3}\left(g_{0} z_{2}-\frac{1}{\tau} q-\dot{\alpha_{q}}\right) .
$$

We will now consider two cases: a) $z_{3} \leq 0$ and b) $z_{3}>0$.

a) $z_{3} \leq 0$. Since $\delta_{a}<0$, we have from (34), which is equivalent to (36) but written in the $z$ coordinates, that

$$
\begin{gathered}
-C_{3} z_{3}<g_{0} z_{2}-\frac{1}{\tau} q-\dot{\alpha}_{q}, \\
z_{3}\left(g_{0} z_{2}-\frac{1}{\tau} q-\dot{\alpha}_{q}\right)<-C_{3} z_{3}^{2} .
\end{gathered}
$$

Thus, we obtain

$$
\dot{U}_{3} \leq-C_{2} z_{2}^{2}-C_{3} z_{3}^{2} .
$$

b) $z_{3}>0$. In this case, we have from (45), by inserting for $\dot{\alpha}_{q}$ and rearranging terms, that

$$
\begin{aligned}
\dot{U}_{3} \leq & -C_{2} z_{2}^{2}-\frac{C_{2}+h_{0}}{4 g_{0}} a_{1}\left(p_{\text {ref }}-b_{0}\right) z_{3} \\
& -\left(\frac{1}{2 \tau}-\left(C_{2}+h_{0}\right)\right) q z_{3} \\
& -q z_{3}\left(\frac{1}{2 \tau}+\frac{C_{2}+h_{0}}{g_{0}} g_{1} z_{2}\right) \\
& -\frac{C_{2}+h_{0}}{4 g_{0}} a_{1} z_{3}\left(4 z_{1}+\left(p_{\text {ref }}-b_{0}\right)\right) \\
& -\frac{C_{2}+h_{0}}{4 g_{0}} z_{3}\left(4 h_{1} z_{2}^{3}+a_{1}\left(p_{\text {ref }}-b_{0}\right)\right) \\
& -\frac{z_{3}}{4 g_{0}}\left(\left(C_{2}+h_{0}\right) a_{1}\left(p_{\text {ref }}-b_{0}\right)\right. \\
& \left.-4\left(g_{0}^{2}+C_{2} h_{0}+h_{0}^{2}\right) z_{2}\right) .
\end{aligned}
$$

Using (37), and imposing the conditions

$$
\begin{aligned}
& z_{1} \geq-\frac{1}{4}\left(p_{\text {ref }}-b_{0}\right) \\
& z_{2} \leq \frac{a_{1}\left(C_{2}+h_{0}\right)\left(p_{\text {ref }}-b_{0}\right)}{4\left(g_{0}^{2}+C_{2} h_{0}+h_{0}^{2}\right)} \\
& z_{2} \geq-\min \left\{\frac{g_{0}}{2 \tau g_{1}\left(C_{2}+h_{0}\right)}, \sqrt[3]{\frac{a_{1}\left(p_{r e f}-b_{0}\right)}{4 h_{1}}}\right\}
\end{aligned}
$$


we obtain

$$
\dot{U}_{3} \leq-C_{2} z_{2}^{2}-\frac{C_{2}+h_{0}}{4 g_{0}} a_{1}\left(p_{r e f}-b_{0}\right)\left|z_{3}\right| .
$$

In view of (42),(48) and (53), LaSalle's invariance principle can be invoked as in the first part of this proof to establish asymptotic stability of $z=0$ and that initial conditions satisfying (50)-(52) are contained in the region of attraction of $z=0$. Finally, we note that the conditions (50)-(52), written in terms of $(p, w, q)$, exactly characterize the set $\mathcal{A}$, as given by (38)-(41).

\section{Simulation results}

In this section we test our proposed backstepping controller on model (1)-(2). For simulation studies, the following values are selected as "true" parameters for the system: $h_{0}=1, h_{1}=50, g_{0}=0.125, g_{1}=5$, $a_{1}=0.025, b_{0}=3.5, \tau=0.1$, and the pressure set point $p_{\text {ref }}=3.51$. The design objective is to stabilize $p$ at the desired set point $p_{\text {ref }}$.

With the proposed backstepping controller, we take the following set of design parameters: $C_{2}=0.2$ and $C_{3}=$ 5. The initials are set as $p(0)=3.51, w(0)=q(0)=0$ and $u_{0}=[0.10,0.90]$, respectively. Figure 3 illustrates the backstepping controller applied for stabilization in the unstable region at reference pressure $p_{\text {ref }}=3.51$. Figure 4 shows that the system looses stability at the pressure $p_{\text {ref }}=3.49$, which is below the point $p=b_{0}=3.5$. The simulation results verify our theoretical findings.
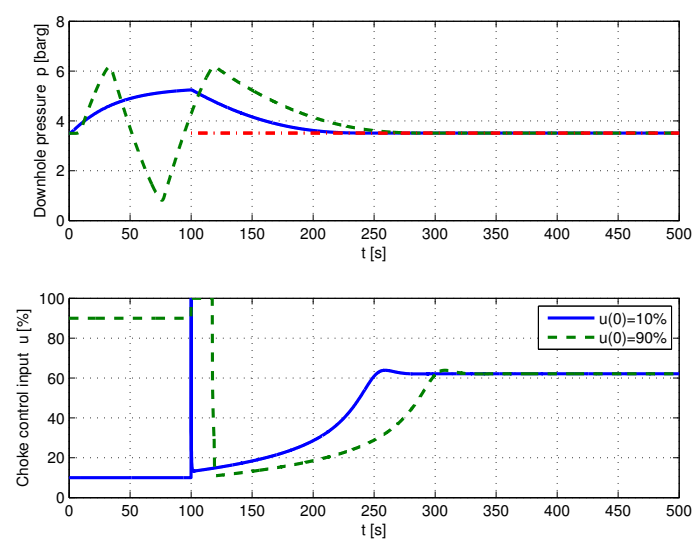

Figure 3: Simulations illustrating stabilization in the unstable region using backstepping.
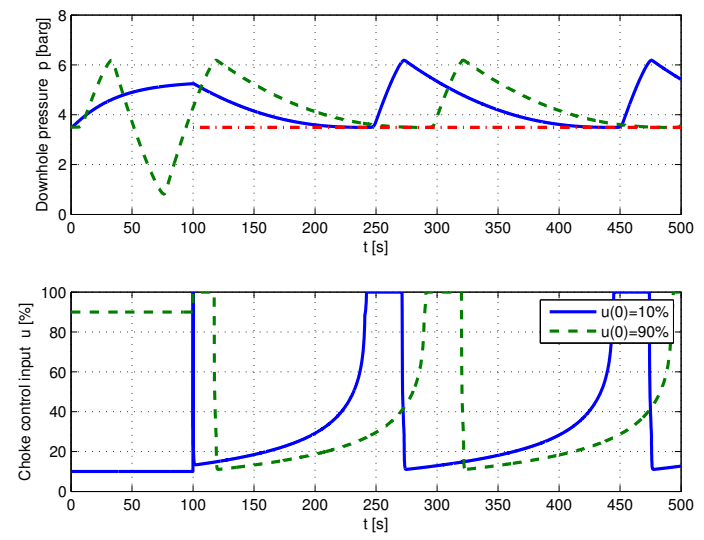

Figure 4: Simulations of an attempt to stabilize at $p_{\text {ref }}=3.49$, which is below what is physically feasible.

\subsection{PI control}

The conventional way to stabilize riser slugging is by applying a simple control law $u_{P I}$ of the form

$$
u_{P I}=u_{I}-K_{p}\left(p-p_{r e f}\right),
$$

where $u_{I}$ is the bias for a given pressure set-point $p_{\text {ref }}$, generated by slow integral action according to

$$
\dot{u}_{I}=-\frac{K_{i}}{T_{i}}\left(p-p_{r e f}\right) .
$$

By linearizing the closed loop dynamics, the Jocabian matrix is

$$
A=\left[\begin{array}{ccc}
0 & 1 & 0 \\
-a_{1} & h_{0}-g_{1} q_{r e f} & g_{0} \\
-\frac{K_{p}}{\tau} \delta^{\prime}\left(u_{I}\right) & 0 & -\frac{1}{\tau}
\end{array}\right],
$$

which has the characteristic equation

$$
\begin{aligned}
& \lambda^{3}+\left(\frac{1}{\tau}-h_{0}+g_{1} q_{r e f}\right) \lambda^{2} \\
& +\frac{1}{\tau}\left(\tau a_{1}-h_{0}+g_{1} q_{r e f}\right) \lambda+\frac{g_{0}}{\tau} \delta^{\prime}\left(u_{I}\right) K_{p}+\frac{a_{1}}{\tau}=0 .
\end{aligned}
$$

According to the Hurwitz criterion, it turns out that local exponential stability can be achieved by PI control if

$$
p_{\text {ref }}>b_{0}+\frac{h_{0} g_{0}}{a_{1} g_{1}}-\min \left\{\frac{g_{0} \tau}{g_{1}}, \frac{g_{0}}{g_{1} \tau a_{1}}\right\}
$$


and $K_{p}$ is given by

$$
\underline{K}_{p}<K_{p}<\bar{K}_{p}
$$

where

$$
\begin{aligned}
\underline{K}_{p} & =\frac{\left(\frac{1}{\tau}-h_{0}+g_{1} q_{\text {ref }}\right)\left(\tau a_{1}-h_{0}+g_{1} q_{\text {ref }}\right)-a_{1}}{\delta^{\prime}\left(u_{I}\right) g_{0}} \\
\bar{K}_{p} & =\frac{-a_{1}}{g_{0} \delta^{\prime}\left(u_{I}\right)}, \\
q_{\text {ref }} & =\frac{a_{1}}{g_{0}}\left(p_{\text {ref }}-b_{0}\right) .
\end{aligned}
$$

Here, we have treated $u_{I}$ as constant, corresponding to the choke opening at the equilibrium $\left(p_{\text {ref }}, 0, a_{1}\left(p_{\text {ref }}-\right.\right.$ $\left.\left.b_{0}\right) / g_{0}\right)$. The bifurcation point corresponds to

$$
p_{\text {ref }}=b_{0}+\frac{h_{0} g_{0}}{a_{1} g_{1}} .
$$

Figure 5 illustrates PI controller applied for stabilization in the unstable region at reference pressure $p_{\text {ref }}=4.498$. Figure 6 shows that the system looses stability at the pressure $p_{\text {ref }}=4.45$, which is below the required reference $p_{\text {ref }}>4.4975$. The bifurcation point corresponds to $p_{\text {bifur }}=4.5$. The design parameters are chosen as $K_{p}=0.1, K_{i}=0.1$ and $T_{i}=25$.
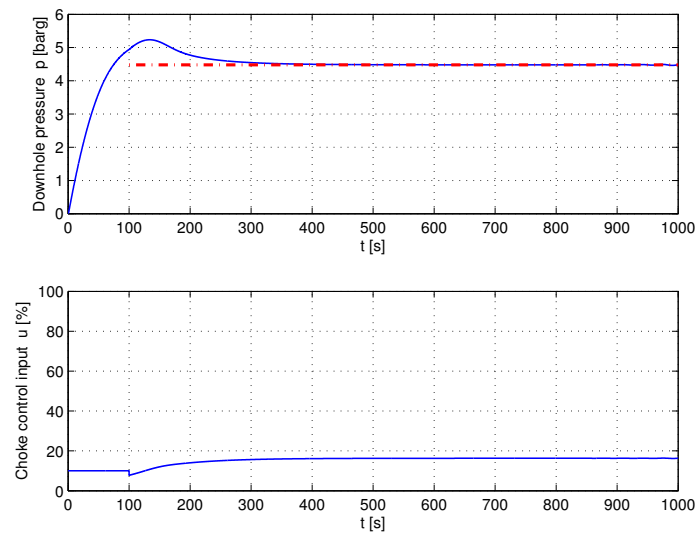

Figure 5: Simulations of PI stabilization at a pressure in the unstable region $p_{\text {ref }}=4.498$ using PI controller.
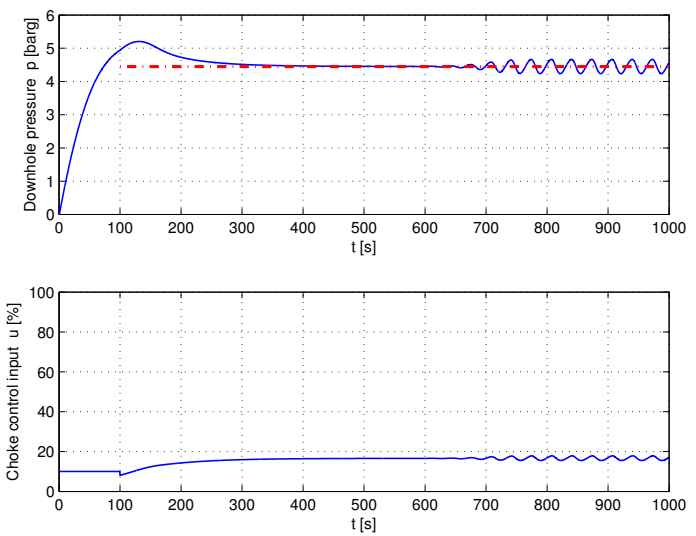

Figure 6: Simulations of an attempt to stabilize at a pressure in the unstable region $p_{\text {ref }}=4.45$ using PI controller.

\subsection{PD control}

Another way to stabilize riser slugging is by applying a simple control law $u_{P D}$ of the form

$$
u_{P D}=u_{I}+u_{D}-K_{p}\left(p-p_{r e f}\right),
$$

where $u_{I}$ is the bias for a given pressure set-point $p_{\text {ref }}$, and $u_{D}$ is the derivative action according to

$$
u_{D}=-K_{d} \frac{d\left(p-p_{r e f}\right)}{d t}=-K_{d} w .
$$

By linearizing the closed loop dynamics, the Jocabian matrix is

$$
A=\left[\begin{array}{ccc}
0 & 1 & 0 \\
-a_{1} & h_{0}-g_{1} q_{r e f} & g_{0} \\
-\frac{K_{p}}{\tau} \delta^{\prime}\left(u_{I}\right) & -\frac{K_{d}}{\tau} \delta^{\prime}\left(u_{I}\right) & -\frac{1}{\tau}
\end{array}\right],
$$

which has the characteristic equation

$$
\begin{aligned}
& \lambda^{3}+\left(\frac{1}{\tau}-h_{0}+g_{1} q_{\text {ref }}\right) \lambda^{2}+\left(\frac{g_{0}}{\tau} \delta^{\prime}\left(u_{I}\right) K_{p}+\frac{a_{1}}{\tau}\right) \\
& +\frac{1}{\tau}\left(\tau a_{1}-h_{0}+g_{1} q_{\text {ref }}+K_{d} g_{0} \delta^{\prime}\left(u_{I}\right)\right) \lambda=0 .
\end{aligned}
$$

According to the Hurwitz criterion, it turns out that local exponential stability can be achieved by PD control if

$p_{\text {ref }}>b_{0}+\frac{h_{0} g_{0}}{a_{1} g_{1}}-\min \left\{\frac{g_{0} \tau}{g_{1}}+\frac{g_{0}^{2} K_{d}}{g_{1} a_{1}} \delta^{\prime}\left(u_{I}\right), \frac{g_{0}}{g_{1} \tau a_{1}}\right\}$, 
$K_{d}$ satisfies

$K_{d}<\frac{g_{0} \delta^{\prime}\left(u_{I}\right) K_{p}+a_{1}}{\left(\frac{1}{\tau}-h_{0}+g_{1} q_{r e f}\right) g_{0} \delta^{\prime}\left(u_{I}\right)}-\frac{\tau a_{1}-h_{0}+g_{1} q_{r e f}}{\delta^{\prime}\left(u_{I}\right) g_{0}}$,

and $K_{p}$ satisfies

$$
\underline{K}_{p}<K_{p}<\bar{K}_{p}
$$

where

$$
\begin{aligned}
\underline{K}_{p}= & \frac{\left(\frac{1}{\tau}-h_{0}+g_{1} q_{\text {ref }}\right)\left(\tau a_{1}-h_{0}+g_{1} q_{\text {ref }}\right)-a_{1}}{\delta^{\prime}\left(u_{I}\right) g_{0}} \\
& +K_{d}\left(\frac{1}{\tau}-h_{0}+g_{1} q_{\text {ref }}\right), \\
\bar{K}_{p}= & \frac{-a_{1}}{g_{0} \delta^{\prime}\left(u_{I}\right)}, \\
q_{\text {ref }}= & \frac{a_{1}}{g_{0}}\left(p_{\text {ref }}-b_{0}\right) .
\end{aligned}
$$

Figure 7 illustrates PD controller applied for stabilization at reference pressure $p_{\text {ref }}=4.6$. The design parameters are chosen as $K_{p}=5$ and $K_{d}=5$, which satisfy the stability conditions. Figure 8 shows that the system looses stability at the pressure $p_{\text {ref }}=3.51$. The design parameters are chosen as $K_{p}=0.02$ and $K_{d}=-1$.
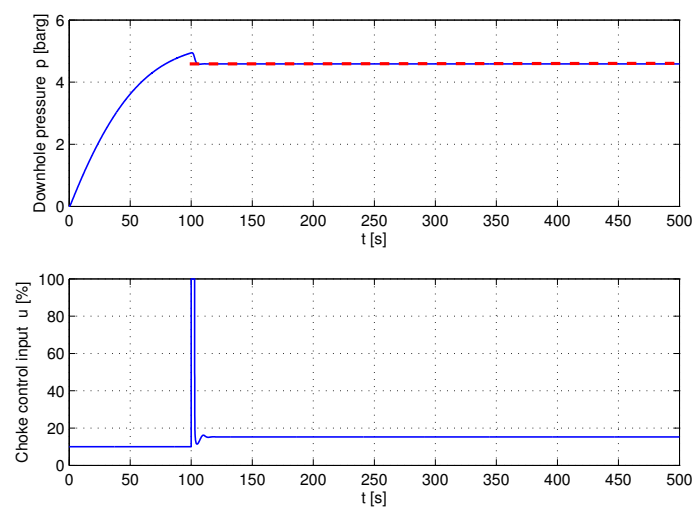

Figure 7: Simulations of PD stabilization at a pressure $p_{\text {ref }}=4.60$ using PD controller.

\section{Conclusion}

This paper illustrates the potential of nonlinear modelbased control applied for stabilization of unstable flow in oil wells. A simple empirical model is developed that describes the qualitative behavior of the downhole pressure in case of severe riser slugging. Two con-
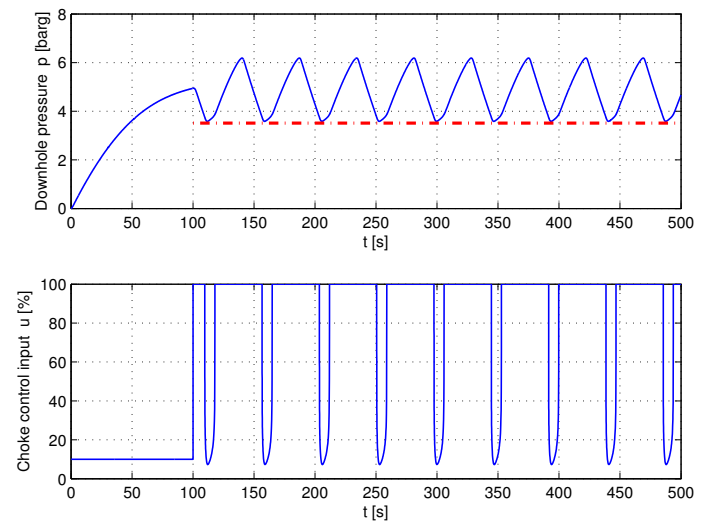

Figure 8: Simulations of an attempt to stabilize at $p_{\text {ref }}=3.51$ using $\mathrm{PD}$ controller.

trol schemes are developed using the integrator backstepping approach. The first scheme is an exact cancelling design because we simply cancel existing dynamics including some stabilizing nonlinearities, which may waste control effort and make the control law complicated. To avoid cancellation of useful nonlinearities, a better controller is developed, which in addition takes input saturation into account. It is shown that the proposed backstepping control scheme can guarantee asymptotic stability of the closed-loop system with saturated control. The proposed backstepping controller can stabilize at lower pressure setpoints, corresponding to higher flow rates, than PI and PD controllers. When the pressure setpoint is low, parameters of the PD controller that are feasible according to the Hurwitz criterium, give a very aggressive actuation causing the choke to saturate repeatedly and stabilization is not achieved. For the same pressure setpoint, the proposed backstepping controller is easy to tune. Simulation results are presented to illustrate the performance of the proposed control scheme.

\section{References}

Bøe, A. Severe slugging characteristics ; part i:flow regime for severe slugging ; part ii: Point model simulation study. Presented at Selected Topics in Two-Phase Flow, NTH, Trondheim, Norway, 1986.

Dalsmo, M., Halvorsen, E., and Slupphaug, O. Active feedback control of unstable wells at the brage field. SPE Annual Technical Conference and Exhibition, San Antonio, Texas, September 29-October 2, SPE 77650, 2002. 
Drengstig, T. and Magndal, S. Slugcontrol of production pipeline. In Proceedings of SIMS2001, Porsgrunn, Norway. 2002 pages 361-366.

Eikrem, G. Stabilization of gas-lift wells by feedback control. Ph.D. thesis, Norwegian University of Science and Technology, 2006.

Farghaly, M. Study of severe slugging in real offshore pipeline riser-pipe system. SPE Journal, SPE 15726, 1987.

Godhavn, J.-M., Fard, M., and Fucks, P. New slug control strategies, tuning rules and experimental results. Journal of process control, 2005. 15:454-463.

Henriot, V., Courbot, A., Heintz, E., and Moyeux, L. Simulation of process to control severe slugging: Application to dunbar pipeline. SPE Annual Technical Conference and Exhibition. Houston, Texas, 3-6 October, SPE 56461, 1999.

Jansen, B., Dalsmo, M., Nkkelberg, L., Havre, K., Kristiansen, V., and Lemetayer, P. Automatic control of unstable gas lifted wells. SPE Annual Technical Conference and Exhibition, Houston, Texas, Oct. 3-6, SPE 56832, 1999.

Khalil, H. K. Nonlinear Systems. 3rd ed, Prentice-Hall, U.S., 2002.

Kinvig, J. and Molyneux, P. Slugging control. US patent US6716268, 2001.

Kovalev, K., Cruickshank, A., and Purvis, J. The slug suppression system in operation. SPE Journal, SPE 84947,2003

Kovalev, K., Seelen, M., and Haandrikman, G. Vesselless S3: advanced solution to slugging pipelines. SPE Journal, SPE 88569, 2004.

Lin, P. Y. and Hanratty, T. J. Prediction of the initiation of slugs with linear stability theory. Int. J. Multiphase Flow, 1986. 12:79-98.

Molyneux, P., Tait, A., and Kinvig, J. Characterisation and active control of slugging in a vertical riser. In Proceedings from BHR Group Conference: multiphase technology. 2000 .

Pickering, P., Hewitt, G., Watson, M., and Hale, C. The prediction of flows in production risers - truth \& myth? In IIR Conference. 2001.

Schmidt, Z., Brill, J., and Beggs, H. Experimental study of severe slugging in a two-phase flow pipelineriser system. SPE Journal, SPE 8306, 1980. pages 407-414.
Schmidt, Z., Doty, D., and Dutta-roy, K. Severe slugging in offshore pipeline riser-pipe systems. $S P E J$ SPE 1985, 1985.

Sinègre, L. Dynamic study of unstable phenomena stepping in gas-lift activated wells. Ph.D. thesis, Mines Paris, 2006.

Storkaas, E. Stabilizing control and controllability: Control solutions to avoid slug flow in pipeline. Ph.D. thesis, Norwegian University of Science and Technology, 2005.

Storkaas, E., Skogestad, S., and Godhavn, J.-. A lowdimensional dynamic model of severe slugging for control design and analysis. In Presented at Multiphase'03, San Remo, Italy, 11-13. 2003.

Taitel, T. Stability of severe slugging. Int. J. Multiphase flow, 1986. 12(2):203-217.

Taitel, T. and Dukler, A. A model for flow regime transition in horizontal and near horizontal gas-liquid flows. Aiche J., 1975. 19(3):47-55.

Tengesdal, J., Sarica, C., and Thompson, L. Severe slugging attenuation for deepwater multiphase pipeline and riser systems. SPE Production and $\mathrm{Fa}$ cilities, 2003. 18(4):269-279.

Wilkins, R., Glassmeyer, S., Rosebrock, G., Storage, K., and Storage, T. Surfactant use for slug flow pattern suppression in horizontal pipe. In Proceedings of the 8th International Symposiom on Gas-Liquid Flows: ASME/JSME Joint Fluids Engineering Division Summer Meeting, July 6-10. 2003 .

Zakarian, E. Analysis of two-phase flow instabilities in pipe-riser systems. In Proceedings of PVP 2000, July 23-27, Seattle, USA. 2000 . 